\title{
ELABORAÇÃO DE OFICINA HOSPITALAR PARA PREVENÇÃO DE HIPOTERMIA DO RECÉM-NASCIDO LOGO APÓS O NASCIMENTO
}

\author{
AUTOR: Isayama, Ricardo Noboro (Isayama, RN) \\ ORIENTADOR: de Lima, Renato Oliveira (de Lima, RO) \\ INSTITUIÇÃO: Centro Universitário São Camilo - Campus Ipiranga, São Paulo-SP
}

INTRODUÇÃO:

A hipotermia do recém-nascido $(\mathrm{RN})$ é um dos principais fatores de risco de morbimortalidade neonatal, estando relacionada à síndrome do desconforto respiratório, hipoglicemia, icterícia, hemorragia intraventricular, enterocolite necrotizante, e parada cardiorrespiratória (CHEBBOUT et al, 2017; PINHEIRO, 2018; HARER et al., 2017; NOSHERWAN et al, 2017; MC CALL et al, 2018; NECZYPOR et al, 2017; WYCKOFF, 2014; O'DONNELL, 2012; RAGHUVEER \& COX, 2011; BISSINGER \& ANNIBALE, 2010; TE PAS et al., 2010; KNOBEL et al, 2005; NIERMEYER et al, 2000; KUMAR \& AGGARWAL, 1998; MAIMBOLWA et al, 1997). Sabe-se que o risco de hipotermia neonatal é maior no primeiro minuto de vida, conhecido como o "minuto de ouro" ou Gold minute da língua inglesa (NECZYPOR \& HOLLEY, 2017; WYCKOFF, 2014; BISSINGER \& ANNIBALE, 2010). Devido à imaturidade da termorregulação do $\mathrm{RN}$, profissionais de saúde devem prevenir a hipotermia, mantendo-se a temperatura entre $36,5^{\circ} \mathrm{C}$ e $37,5^{\circ} \mathrm{C}$.

\section{METODOLOGIA:}

Inicialmente, a oficina é divulgada aos profissionais de saúde no centro obstétrico, na UTI neonatal, nas enfermarias ou pronto socorro, dentre outros. Espaços e equipamentos do próprio hospital serão utilizados, e as atividades serão orientadas por preceptor neonatologista e por acadêmicos de medicina. Um circuito de 60 minutos começará com uma apresentação expositiva de 10-20 minutos sobre o tema. Em seguida, os participantes são divididos em grupos, rodiziando a cada 10 minutos nas seguintes estações: 1) planejamento e técnicas no centro obstétrico, 2) uso da incubadora de transporte, 3) transporte intra-hospitalar, e 4) admissão do RN e cuidados na UTI neonatal. A oficina pode ocorrer em turnos para flexibilizar a capacitação entre os profissionais. Os protocolos de padronização das medidas preventivas são aqueles estabelecidos pelas diretrizes internacionais sobre prevenção de hipotermia do recém nascido (HARER et al, 2017; MC CALL et al, 2018; O'DONNELL, 2012; NIERMEYER et al, 2000).

\section{OBJETIVO:}

Elaborar oficina hospitalar aos profissionais de saúde para prevenção de hipotermia do RN logo após o nascimento.

\section{RESULTADOS:}

A oficina hospitalar poderá capacitar profissionais de saúde no cuidado ao RN logo após o nascimento. Com isto, prevenir-se-á a hipotermia do $\mathrm{RN}$ e poderá disseminar este conhecimento ainda negligenciado por muitos. Tais atividades de educação continuada serão orientadas por preceptor neonatologista e acadêmicos de medicina, favorecendo a interação com a equipe multiprofissional. Os temas abordados incluirão monitoramento térmico do binômio mãe-filho, uso adequado do berço de reanimação, filme plástico e dupla touca em 
$\mathrm{RNs}<34$ semanas, uso de colchão térmico nos RNs $<1000 \mathrm{~g}$ e, o transporte e admissão do RN em UTI neonatal. A sorientaçoes especificas de cada estação são apresentadas em cartazes, conforme figuras abaixo.

\section{ESTAÇÃO I}

\section{PREPARANDO A SALA DE PARTO PARA A PREVENÇÃO DA HIPOTERMIA DO RN}

- Manter a temperatura da sala de parto entre $23^{\circ} \mathrm{C}$ a $26^{\circ} \mathrm{C}$ com termômetros de ambiente;

- Manter incubadora de transporte em $37^{\circ} \mathrm{C}$;

- Manter berço de reanimação permanentemente ligado com radiância de $30 \%$ com aumento até $90 \%$, no momento próximo ao nascimento;

- Separar previamente: saco plástico poroso $(40 \mathrm{~cm}$ x $60 \mathrm{~cm}$ ) com recorte para a passagem da cabeça (triângulo plástico), touca, campos e compressas estéreis aquecidos no berço de reanimação; - Utilizar o triângulo para a prática da "dupla touca"; - Solicitar colchão térmico químico para RN menores de 34 semanas. 


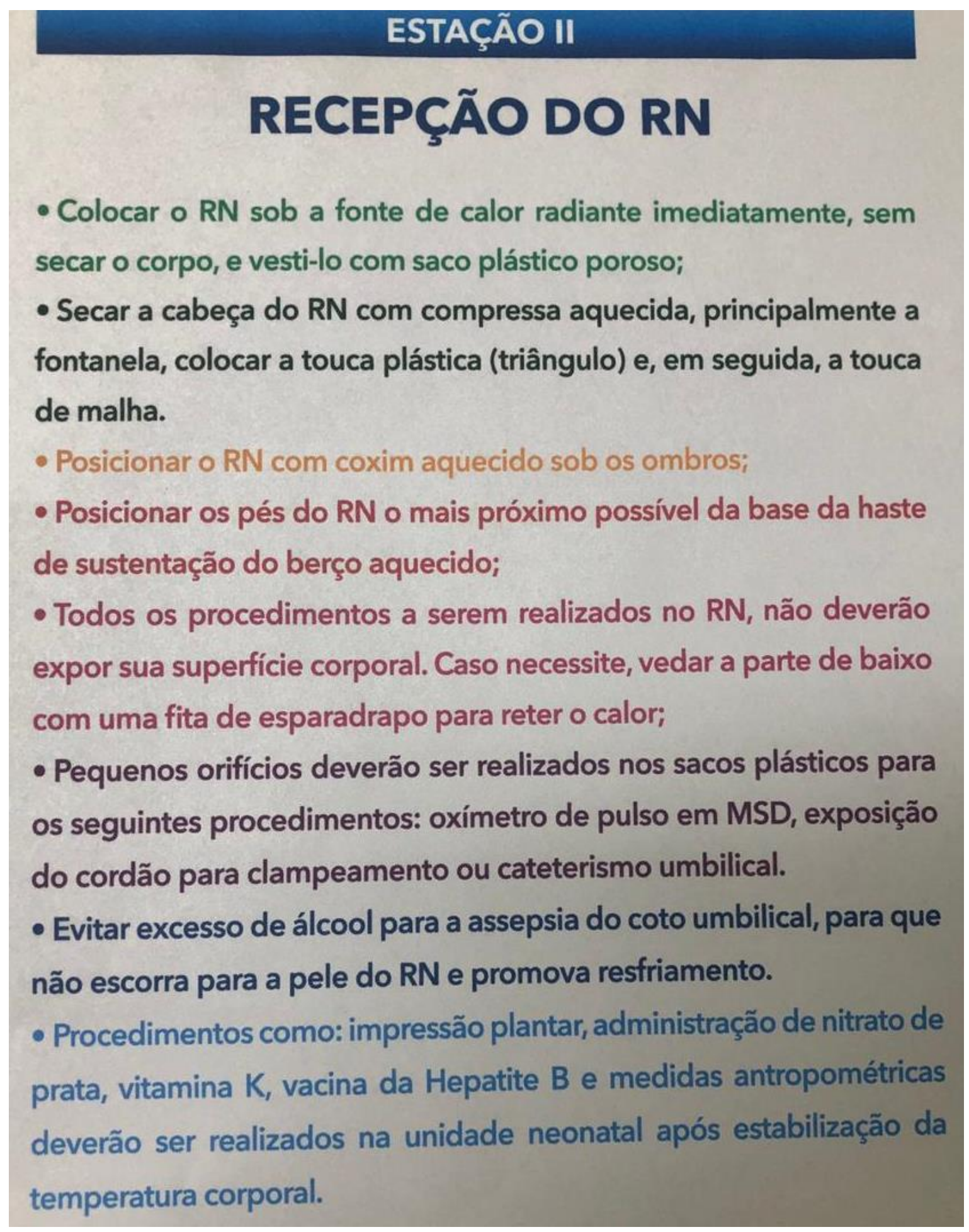




\section{ESTAÇÃO III}

\section{TRANSPORTE DO RN}

- Após a estabilização do RN, transportá-lo em incubadora de transporte devidamente aquecida, abrir a incubadora somente no momento imediato ao início do transporte;

- Manter temperatura da incubadora em $37^{\circ} \mathrm{C}$;

- Não esqueça do cinto de segurança da incubadora:

$\Rightarrow$ Para RN maior de 1.500 gramas, utilize 2 cintos (tórax e pernas);

$\Rightarrow$ Para RN menor de 1.500 gramas, utilize 1 cinto no tórax - Mantenha o saco plástico e colchão térmico durante todo o transporte;

- Coloque a cabeça do RN voltada para o lado da abertura lateral da incubadora de transporte;

- Sempre que a incubadora estiver parada, deixar os rodízios travados;

- Evite estresse auditivo para o RN: feche as portinholas com delicadeza, evite atritos na superfície da incubadora de transporte,

- Não permita que a incubadora esbarre em paredes e portas. 


\section{RECEPCIONANDO O RN NA UTIN}

- Na unidade neonatal, medir a temperatura imediatamente a sua chegada.

- Somente retirar o saco plástico após temperatura igual ou superior a $36,5^{\circ} \mathrm{C}$.

- O RN evacuou? Urinou? Está sujo de sangue? Não tem importância! Se ainda estiver hipotérmico, mantenha-o envolvido no saco plástico.

- Só abra a incubadora da UTI no momento imediato a entrada do RN.

- Desligue o ar-condicionado da sala. - Priorize a MANIPULAÇÃO MíNIMA nas primeiras horas.

CONCLUSÃO:

Conclui-se que a elaboração de oficina hospitalar poderá prevenir a hipotermia neonatal logo após o nascimento, melhorando o prognóstico do $\mathrm{RN}$, aumentando a qualidade do serviço hospitalar, favorecendo a integração do hospital com as atividades acadêmicas do curso de medicina, e promovendo a disseminação do conhecimento técnico em âmbito multiprofissional. 
DESCRITORES: oficina, hipotermia, neonatal, RN, hospital

\section{REFERENCIAS:}

BISSINGER, RL; ANNIBALE, DJ. Thermoregulation in very low-birth-weight infants during the golden hour: results and implications.Adv Neonatal Care., v.10, n.5, p. 230-8, 2010.

CHEBBOUT, R; NEWTON, RS; WALTERS, M; WRENCH, IJ; WOOLNOUGH, M. Does the addition of active body warming to in-line intravenous fluid warming prevent maternal hypothermia during elective caesarean section? A randomised controlled trial. Int $\mathrm{J}$ Obstet Anesth., n.31, p.37-44, 2017.

HARER, MW; VERGALES, B; CADY, T; EARLY, A; CHISHOLM, C; SWANSON, JR. Implementation of a multidisciplinary guideline improves preterm infant admission temperatures. J Perinatol., v.37, n.11, p.1242-47, 2017.

KNOBEL, RB; VOHRA, S; LEHMANN, CU. Heat loss prevention in the delivery room for preterm infants: a national survey of newborn intensive care units. J Perinatol., v.25, n.8, p.5148, 2005.

KNOBEL, RB; WIMMER, JE; JR HOLBERT, D. Heat loss prevention for preterm infants in the delivery room. J Perinatol., v.25, n.5, p.304-8, 2005.

KUMAR, R; AGGARWAL, AK. Body temperatures of home delivered newborns in north India. Trop Doct., v.28, n.3, p.134-6, 1998.

MAIMBOLWA, MC; RANSJÖ-ARVIDSON, AB; NG'ANDU, N; SIKAZWE, N; DIWAN, VK. Routine care of women experiencing normal deliveries in Zambian maternity wards: a pilot study. Midwifery., v.13, n.3, p.125-31, 1997.

MC CALL, EM; ALDERDICE, F; HALLIDAY, HL; VOHRA, S; JOHNSTON, L. Interventions to prevent hypothermia at birth in preterm and/or low birth weight infants. Cochrane Database Syst Rev. v.12, n.2, In:CD004210, 2018.

NECZYPOR, JL; HOLLEY, SL. Providing Evidence-Based Care During the Golden Hour. Nurs Womens Health., v.21, n.6, p.462-472, 2017.

NIERMEYER, S; KATTWINKEL, J; VAN REEMPTS, P; NADKARNI, V; PHILLIPS, B; ZIDEMAN, D; AZZOPARDI, D; BERG, R; BOYLE, D; BOYLE, R; BURCHFIELD, D; CARLO, W; CHAMEIDES, L; DENSON, S; FALLAT, M; GERARDI, M; GUNN, A; HAZINSKI, MF; KEENAN, W; KNAEBEL, S; MILNER, A; PERLMAN, J; SAUGSTAD, OD; SCHLEIEN, C; SOLIMANO, A; SPEER, M; TOCE, S; WISWELL, T; ZARITSKY. International Guidelines for Neonatal Resuscitation: An excerpt from the Guidelines 2000 for Cardiopulmonary Resuscitation and Emergency Cardiovascular Care: International Consensus on Science. Contributors and Reviewers for the Neonatal Resuscitation Guidelines. A.Pediatrics., v.106, n.3, p.E29, 2000.

NOSHERWAN, A; CHEUNG, PY; SCHMÖLZER, GM. Management of Extremely Low Birth Weight Infants in Delivery Room. Clin Perinatol., v.44, n.2, p.361-375, 2017.

O'DONNELL, CP. Turn and face the strange - ch..ch..ch..changes to neonatal resuscitation guidelines in the past decade. J Paediatr Child Health., v.48, n.9, p.735-9, 2012.

PINHEIRO, JMB. Prevenção de hipotermia em recém-nascidos prematuros - princípios simples para uma tarefa complicada, J. Pediatr., v.94, n.4, 2018.

RAGHUVEER, TS; COX, AJ. Neonatal resuscitation: an update. Am Fam Physician. v.83, n.8, p.911-8, 2011.

TE PAS, AB; LOPRIORE, E; DITO, I; MORLEY, CJ; WALTHER, FJ. Humidified and heated air during stabilization at birth improves temperature in preterm infants. Pediatrics., v.125, n.6), p.e1427-32, 2010.

WYCKOFF, MH. Initial resuscitation and stabilization of the periviable neonate: the Golden-Hour approach. Semin Perinatol., v.38, n.1, p.12-6, 2014. 